\title{
Algunas consideraciones sobre el impacto sociocultural de las nuevas tecnologías de la información y las comunicaciones
}

Some considerations on the socio cultural impact of the new information and communication technologies

\author{
Alfredo Fernández Rodríguez \\ Lilliam Alvarez Díaz
}

\section{Resumen}

Del uso de Nuevas Tecnologías de la Información las Comunicaciones, NTIC, han emergido funciones no previstas por sus creadores. Pues en unos primeros momentos estas solo fueron concebidas con un carácter instrumental, o sea, fueron creadas para las estrictas funciones de: informar, comunicar y entretener. El presente trabajo abordará algunas de las nuevas funciones, no previstas, que han surgido como resultado del uso e interacción social entre los usuarios de estas nuevas tecnologías. Se abordarán en particular, las tecnologías de la telefonía celular, la Internet, la componente social y el impacto de las NTIC en la comunicación social. Definiciones sobre lo instrumental y lo no instrumental de las NTIC se abordarán y discutirán, aportando nuevas consideraciones a los estudios socioculturales de estas tecnologías contemporáneas.

Palabras claves: Nuevas tecnologías de la información y las comunicaciones, impacto sociocultural de las NTIC, función no instrumental de las NTIC, influencia de las NTIC en la comunicación social.

\begin{abstract}
New aspects non thought by the original authors have emerged from the New Information and Communication Technologies, NICTs, At first these technologies were created for only instrumental functions, that means, to communicate, inform and entertainment. The present work describes some other different aspects that have appeared as a product of the social interactions of the users of these new technologies. In particular, we discuss the use of mobile telephones, internet and social component and impact of the NICTs. Some definitions and concepts about the instrumental and non instrumental functions of the NICTs are presented contributing to enlightening new considerations to the socio cultural studies of these contemporary technologies.
\end{abstract}

Keywords: New Information and Communication Technologies, NICTs, socio cultural impacts of the NICTs, non instrumental functions of the NICTs, influences of the NICTs in thje social communication.

\section{Introducción}

Como resultado de los avances tecnológicos de la contemporaneidad las personas poseen teléfonos celulares, computadoras, DVD, memorias USB, Internet, etc. etc., y a partir de ahí la sociedad tiene, un mayor acceso a la información. Ese nivel de información sería razón suficiente para tener en cuenta a las nuevas tecnologías de la información y las comunicaciones (NTIC) en las investigaciones de comunicación social. Sobre todo porque la utilización de las NTIC brindan también una nueva manera de estar informado, una manera más autónoma de saber. Esa autonomía singulariza la información que se alcanza mediante la tecnología.

Pero esa singularidad comunicativa contemporánea, resultado del uso de las NTIC, es solo una arista de la situación. Para un sociólogo, un comunicador social o un psicólogo le resultaría más importante preguntarse si las NTIC, (al proporcionar un cambio tan violento en el acceso de información del individuo), tienen influencia en la construcción de nuevos sentidos y significados de vida. Dicho en otras palabras, las NTIC no solo sirven para entretener, comunicar e informar, única condición con las que fueron concebidas en su carácter instrumental, sino, que son eso y también más que eso.

Por ejemplo, se sabe que el sentimiento de soledad está relacionado con la idea de no formar parte de algo, la idea de no estar incluido en ningún proyecto de otro, etc. Entonces si este sociólogo, comunicador social o psicólogo, tiene en cuenta al abordar su trabajo que la tecnología nos hace sentirnos menos solos; entonces ahí está una mirada no instrumental de la tecnología aunque el 
científico social se encuentre investigando o no en esa dirección.

El presente trabajo aborda a dos de los integrantes más significativos de las NTIC, la telefonía celular e Internet, para luego brindar argumentos de por qué éstos son más que simples instrumentos de uso y consumo.

Se hace imprescindible, para demostrar que las NTIC son más que instrumentos, una palabra para entenderla en sus dos aristas: como instrumento y como símbolo. Esa necesidad surge por el hecho de que cuando se dice "nuevas tecnologías de la información y las comunicaciones" solo se piensa en que entretiene, comunica y da conocimientos.

Este artículo se auxilia de una propuesta de término que servirá para evitar ambigüedades. Ese término lo denotaremos por la frase sustantivada "Lo tecnológico" y será definido al final del trabajo.

Se profundiza en las componentes social y cultural, generalmente no visible y estudiada en la utilización de las NTIC. Se establece la diferencia y se separa con precisión el empleo pragmático de estas tecnologías dirigidas a informar, a buscar conocimientos, investigar, entretener; de lo sicológico, emocional, social, cultural, discutiendo cuánto se ha transformado nuestro accionar en los ámbitos laborales, sociales y familiares mediante el empleo de estas tecnologías que han ido y seguirán dirigiéndose mucho mas lejos de "esa utopía soñada" que podríamos imaginar, si nos informamos hacia dónde se dirige la Nanoelectrónica y las Nanotecnologías hacia la miniaturización y potenciación cada vez mas de los dispositivos. Por supuesto en el artículo estaremos discutiendo de las NTICs para humanizar y defender la vida y no para la carrera armamentista.

\section{La telefonía celular}

La importancia instrumental del teléfono celular es incuestionable. Tal es así que la literatura especializada lo llama "metadispositivo digital", justamente por ser integrador de otros dispositivos, a saber: el teléfono propiamente dicho, cámara fotográfica, reproductor de música, consola de juegos, etc. Pero es, además, otro aspecto del teléfono celular lo que es de interés en este trabajo. Se trata de su condición de estar estrechamente ligado a la persona, al ser humano. Esa condición permite estudiarlo desde su influencia en la construcción de la identidad del individuo y en la producción de significados sociales que su uso genera.

El intercambio comunicativo se ha potenciado gracias a la telefonía celular porque la conexión ya no depende del lugar sino de la persona. Si dos personas poseen celular, la conexión es independiente del lugar donde se encuentren. A esa posibilidad de "movimiento" que nos da el celular se le llama "comunicación translocal y conectividad continua"2. De lo interlocal y discreto a lo translocal y continuo. Esa comunicación casi instantánea convierte al teléfono móvil en un complemento del sujeto social. Pero no es la voz lo que privilegia al celular, es su capacidad de trasmitir datos, son los mensajes (SMS) lo que convierten al celular en algo relevante dentro de la Sociedad de la Información. El mensaje, aparte de la seguridad de llegada al destino, también se le suma la discreción (la interferencia es menor que en una conversación), es un conector emocional y también institucional ${ }^{3}$. El celular puede crear una complicidad insospechada que no debe ser ignorada por los científicos sociales.

La telefonía celular sustituye la confrontación cara a cara del individuo por una confrontación virtual, esa que llega por voz o por mensaje. Pero no siempre es así, el celular es, también, un medio para el encuentro ${ }^{4}$. El celular es un facilitador, un posibilitador de encuentros sociales. El mensaje celular es una tecnología segura y discreta que permite el encuentro afectivo pero también la coordinación de encuentros grupales. Es lo que se llama "función de micro-coordinación" 5 del celular en los procesos de socialización en grupos de jóvenes y de no tan jóvenes. Es el celular una herramienta relacional tanto de comunidades afectivas como de comunidades de otros intereses.

Gracias al celular la toma de decisiones en la casa, en el trabajo y entre amigos es coparticipativa. No existen razones, si estamos comunicados por el celular, para que nadie tome decisiones por nosotros en cuestiones en las que estamos involucrados. Esa posibilidad de que siempre se pueda contar con nosotros incrementa la seguridad personal y distribuye responsabilidades. No hay excusa para no contar con unos con otros o para no tener en cuenta al otro $^{6}$. De esa manera el celular democratiza la toma de decisiones a todos los niveles, además de llevarla a tiempo real. Se podría decir que el celular descentraliza y des-normaliza.

Para muchos autores ${ }^{7}$, el celular genera identidad en términos simbólicos y estéticos. Afirman que el celular como metadispositivo digital permite el acceso a contenidos y también posibilita producir y gestionar contenidos vinculados a la vida cotidiana del individuo. El usuario tiene la posibilidad de hacer contactos, de enviar imágenes propias, de hacer anotaciones de algún tema, anotar su propia agenda, precisar y justificar por qué le gusta una música y no otra, etc. etc. Sirve el celular entonces como un dispositivo de registro biográfico donde el usuario produce y gestiona sus propias referencias personales. Todo esto desde lo íntimo, no al estilo facebook. 
Pero el celular no es solo privacidad y creación de autobiografías, el celular también muestra una cara relativa a la participación social. Esa participación puede ser difusa, de forma piramidal donde hay un usuario que se relaciona con muchos aunque éstos no se relaciones entre sí, o puede haber una dinámica de contagio ${ }^{8}$. La dinámica de participación social por medio de mensajes de celulares se conoce en España con el nombre de "pásalo".

La telefonía celular, debido a su menor exigencia de alfabetización (es fácil aprender a usar un celular), su carácter personal, su translocalidad y su conectividad continua, se ha insertado con facilidad en la vida cotidiana y esa inserción ha provocado notables transformaciones en numerosos aspectos de la vida social ${ }^{9}$ : coordinación de roles, coordinación de tiempos, gestión de eventos, permeabilización de las fronteras entre trabajo, familia y ocio, co-participación decisional, distribución de responsabilidades, descentralización y desnormalización de las decisiones y de las estructuras grupales.

Como se observa, la telefonía celular no es sólo un fenómeno tecnológico y económico, es también un fenómeno sociocultural de importancia equivalente a otras tecnologías de la información y comunicación, digna de tenerse en cuenta en toda investigación de comunicación social.

\section{Internet}

La mayoría de los artículos escritos sobre Internet tiene un enfoque eminentemente cuantitativo, pretenden medir cuantas personas tienen acceso a la red o qué sitios son más visitados, sin embargo, esa metodología cuantitativa ha dejado de lado preguntas importantísimas, como por ejemplo: ¿cuáles son las experiencias psicosociales y prácticas culturales generadas por el uso de Internet? Usar Internet es una operación práctica y también una operación interpretativa pues está estructurada como una tecnología de relación que reorganiza y hasta cierto punto re-semantiza los conocimientos. Internet es, sin dudas, un objeto tecnológico y una práctica cultural, una especie de movimiento de transformación que puede afectar las diferentes dimensiones de una comunidad, o un grupo e incluso a toda una sociedad.

A partir de los presupuestos señalados en el párrafo anterior, se acudirá a mostrar algunos de los nuevos significados simbólicos que han emergido del sujeto comunitario a partir de la presencia o no, en su vida, de la Internet y el correo electrónico. Los niveles de acceso, uso y apropiación de la información van a distanciar de manera significativa, al sujeto que acceda y use de manera regular estas tecnologías, del que lo hace de manera irregular o nunca ${ }^{10}$. Esto caracteriza la brecha digital, "los info-ricos" y los "info-pobres", los que acceden y tienen información y los que no la tienen.

\section{La componente social de las NTIC}

En general, se confunde la lógica del ingeniero que diseña y construye los objetos tecnológicos de las NTIC con las lógicas propias de quienes los usan. El ingeniero predice para lo que sirve su tecnología pero las personas usan las NTIC para fines "no previstos" y de formas también "no previstas" por los ingenieros o programadores informáticos. El resultado es una especie de híbrido que combina capacidades técnicas y lógicas sociales de los usuarios ${ }^{11}$. De esa manera, las personas hacen usos no previstos y convierten a las NTIC en objetos compatibles a sus deseos, biografías individuales y referencias socioculturales cotidianas.

\section{- Cohesión familiar}

Usualmente, en las mañanas, los miembros de la familia se desplazan a trabajos, escuelas, hospitales, etc. La única certeza es el momento de salir de la casa, de ahí en adelante todo pudiera ocurrir. Y es entonces cuando el celular se vuelve clave para mantener la cohesión imaginaria y seguridad de la familia. El celular es la manera de estar comunicados con "los de la casa". En la mayoría de los casos no se usa para ampliar la red de conocidos o entablar nuevas relaciones, como sucede con Internet, sino para no perder el contacto con los ya conocidos, con ese circuito de afectos y reconocimientos mutuos que excluye a los otros.

Siempre los padres y los hijos se han comunicado a través del teléfono cuando unos u otros se encuentran fuera de la casa, pero con el celular ha cambiado el sentido de la comunicación. El celular sirve a la familia, a los que se quieren, como un hilo imaginario que une la casa con el exterior, como una forma de contrarrestar la incertidumbre o la inseguridad. No obstante, esa comunicación tiene sentidos diferentes para los padres y para los hijos. Los padres necesitan esa "correa digital" ${ }^{12}$ para que sus hijos estén disponibles y así calmar esa ansiedad de que estén "fuera", aunque también ven 
en Internet como un medio de introducir "extraños" en la casa. Los hijos ven esa "correa" de otra manera. El celular de la madre es símbolo de autoridad y control pues significa el derecho de saber dónde y con quiénes están. El celular representa una extensión del hogar.

\section{- Socialización}

Las tecnologías socializan, específicamente Internet se comporta como un mercado, uno se encuentra con gente conocida, se compran cosas, se obtiene información, se cuenta cuentos y se enteran las personas de lo que sucede en el mundo. Internet socializa porque es un espacio público, entendiendo como público a aquel lugar donde se accede sin permiso y donde no hay segmentación social. Nuevamente la Internet sigue abriendo brechas entre los que la tienen y los que no.

\section{- Información}

Lo que la mayoría de las personas consumen en Internet es información. De índole profesional (artículos, consultar libros, etc.), se buscan aplicaciones diversas que sirven para crear páginas, traducir lenguajes o montar diapositivas. También se bajan películas y música, se consultan carteleras, etc. Pero el valor de esa información no es sólo instrumental en el sentido de que solo sirva para apoyar tareas o entretener; la red contiene todo lo que en las culturas (sobre todo juvenil) se ha vuelto relevante y significativo de compartir con los demás. Esa información se convierte en un objeto de relación con los otros ${ }^{13}$. De la misma manera que muchas personas pasean por el supermercado sin comprar nada, los jóvenes se desplazan por la red, buscando rarezas, ofertas, pero no compran nada, solo acumulan información que convierten en fuente de prestigio para intercambiar con los otros.

\section{- Identidad, visibilidad y trascendencia social}

El sentido de pertenencia e identidad va cambiando paulatinamente y cada vez más tiene que ver con lealtades que no se asocian a la familia ni a la geografía. Muchos jóvenes no piensan desde una cultura única sino desde algo híbrido que parte de su experiencia local pero con carácter transterritorial y multi-temporal ${ }^{14}$.

Los jóvenes se mueven en dos mundos de experiencias diferentes, off line-on line, que no son vividos como antagónicos sino como continuos, convergentes y complementarios. Investigaciones hechas en varios países muestran que comprenden claramente la frontera simbólica que separa esos dos mundos. "Es la habilidad de saber cruzar con mayor o menor voluntad esa frontera, lo que les brinda placer" ${ }^{p}$.

Las investigaciones demuestran que todo lo que se obtiene en la red (información, entretenimiento, competencia informática, nuevas relaciones) se capitaliza y se valida fundamentalmente en las relaciones persona a persona, como manera de reforzar la identidad propia. Todo lo que se adquiere, ocurre y transcurre en Internet toma sentido si se lleva al mundo real.

Las redes sociales y el celular garantizan la visibilidad del individuo y esa visibilidad contribuye a que se le reconozca en el mundo. La importancia de la visibilidad aflora por el hecho de que en la contemporaneidad lo que no puede ser visto en la red, no existe. Una muestra de la veracidad de esa afirmación es el hecho de que los políticos, los movimientos sociales, los famosos, etc. suben a la red disímiles informaciones relacionadas con ellos. Para muchos, el sentimiento de estar aislado y marginado no se produce por la ausencia de personas a su alrededor sino por la desconexión.

\section{- Tiempo y espacio}

Una de las afirmaciones más recurrentes que se encuentra en la literatura consultada acerca de la naturaleza de Internet es que ha empañado el sentido de espacio y tiempo. Escribe Lévy: "Cuando una persona, una colectividad, un acto, una información se virtualiza, se colocan fuera de ahî", se desterritorializan. Una especie de desconexión los separa del espacio físico o geográfico ordinario y de la temporalidad del reloj y del calendario ${ }^{16}$. Algo similar ocurre con el celular pero desde otro ángulo.

En muchas familias los jóvenes poseen Internet y celular y su cuarto funciona como un espacio de sociabilidad a través de su celular y el correo electrónico y el chat. Ya no es necesario salir de la casa para no estar en la casa, ni hay que pedir permiso para comunicarse con los amigos. Ahí hay una trasgresión del espacio y también del tiempo. El uso de la computadora es individual lo cual plantea una distribución y administración del tiempo diferente al tiempo colectivo dedicado al radio y la televisión. Internet y el celular rompen con la capacidad del radio y la televisión para unir la familia.

Antes, el adentro y el afuera no se marcaba tan drásticamente como ahora. Estar cerca de la casa se ejemplificaba con llamar desde la cocina y decirle a todos que el almuerzo ya está listo, la 
escuela quedaba a tres cuadras y al trabajo se llegaba a pie, en 40 o 50 minutos. Ahora todo ha cambiado. Toda la familia trabaja, la ciudad ha crecido, y el tiempo requerido para trasladarse ha aumentado, la delincuencia se ha potenciado. Dadas esas condiciones aparece la necesidad de verificar donde se encuentran nuestros seres queridos y es ahí donde el celular reinventa imaginariamente el hogar.

Se puede preguntar dónde se encuentra la persona, cuándo regresa, si ha tenido algún problema, y también se envía un mensaje preguntando dónde está tal objeto que está perdido, y también preguntaríamos qué quieres comer hoy etc. La inseguridad o la separación obligan a utilizar el celular, el correo electrónico y otros recursos tecnológicos para recrear virtualmente las rutinas familiares y domésticas. De nuevo se redefine el "aquí" y el "ahora". La comunicación familiar ha trascendido los espacios domésticos. Ahora puede producirse en lugares cambiantes y en movimiento rompiendo con las definiciones formales de lo familiar, es decir, lo íntimo y lo privado. Cuando los miembros de una familia se comunican en la calle, el supermercado, la guagua o en la fila del correo, más que un acto de privatización de lo público, ejercen un acto de domesticidad, pues se desconectan de las miradas del exterior y se conectan con el espacio familiar o de la pareja. En el artículo de Aguado y Martínez se le llama a ese comportamiento público, "privacidades nomádicas"17.

\section{Lo instrumental y lo no instrumental: Lo tecnológico}

Para algunos autores, cultura es antónimo de naturaleza, eso significa que todo lo producido por el hombre es cultural. Las computadoras, teléfonos, las redes sociales, etc., forman parte de la cultura humana. Partiendo de esa premisa, una buena aproximación a lo instrumental de la tecnología sería la siguiente: Conjunto de contenidos culturales con sentido práctico.

Lo instrumental es una cultura objetiva caracterizada por el repertorio de habilidades o conocimientos que posee una persona y que de alguna manera le sirve para adaptarse al espacio donde vive. Incluye dominar artefactos tales como el automóvil, las cámaras fotográficas, los celulares, las computadoras y el video, entre otros. Por medio de esos objetos tecnológicos el hombre contemporáneo accede también al conocimiento de instituciones y a la información cultural. Lo instrumental provee al hombre de una cultura objetual que le permite acceder a contenidos de la cultura social en general.

Por otro lado, definir lo no instrumental de la tecnología realmente sería un problema (muchos conceptos son difíciles de definir, como cultura, por ejemplo, y lo que se hace es caracterizarlo de alguna manera). En este caso nos referimos a aspectos no instrumentales de la tecnología, precisados o ya sugeridos. Entre los puntos a destacar de lo no instrumental de las NTIC se citan:

a. Las NTIC ayuda a sentirnos menos solos.

b. Para muchos, la tecnología les ayuda a controlar la incertidumbre e inseguridad contemporánea.

c. Las NTIC neutraliza la dispersión familiar existente en esta época. A muchas personas les refuerza el sentido de pertenencia a la familia, o a algún grupo real o virtual; en otras palabras, las NTIC contribuye al sentirnos incluidos socialmente.

d. Contribuye a disminuir la fragmentación biográfica de la época.

e. La comunicación con el "otro" aumenta y eso nos exorciza en cierta medida del miedo a quienes no conocemos.

f. A muchos las NTIC los independiza de las tradiciones.

g. Pone, a veces, en crisis el papel predominante de los padres en la familia.

h. Sirve para tener localizadas a las personas, contribuyendo de alguna forma a la pérdida de libertad personal.

\section{- Lo tecnológico}

Teniendo en cuenta todas las ideas, observaciones, citas de autores, reflexiones de los autores y bibliografías revisadas, podría definirse a lo tecnológico como aquello que la tecnología tiene de tangible e intangible, de instrumental y no instrumental, de real y de simbólico, de viable y potencial, de material y no material, de concreta y reflexiva y que de manera general podría dividirse en dos partes o funciones:

1. Su aporte al entretenimiento, la información y al conocimiento de quienes las usan.

2. Su papel de herramienta simbólica que penetra en todos los aspectos de la sociedad, poniendo en crisis o reforzando o redefiniendo en ciertos contextos, nociones como el poder, la autoridad, el derecho, el sentimiento de pertenencia, de libertad y hasta el sentido de la 
vida.

El principal propósito de este trabajo no ha sido otro que hallar todas aquellas características intangibles, simbólicas, no materiales y reflexivas que subyacen en las nuevas tecnologías de la información y las comunicaciones (NTIC) y que en este artículo se han resumido con el término lo "no instrumental" de las NTIC.

\section{Conclusiones}

Como una conclusión de interés se podría afirmar que toda investigación de comunicación social contemporánea que no tenga en cuenta, trate o considere aspectos de "lo tecnológico", según se ha abordado y discutido en este trabajo, corre el riesgo de resultar parcial y fragmentaria. Se concluye que "lo tecnológico" tiene por fuerza que ser inherente a toda investigación de comunicación social contemporánea.

Como indica el titulo de este artículo, con este trabajo los autores nos propusimos tomar en cuenta y develar todas aquellas características intangibles, simbólicas, no materiales y reflexivas que subyacen en las nuevas tecnologías de la información y las comunicaciones, que en el trabajo se han condensado con el término lo "no instrumental" de las NTIC y que también son correlativas a las tradicionales funciones instrumentales de comunicar, entretener e informar.

Para constatar la utilidad práctica del término "lo tecnológico" en investigaciones de comunicación social, se ilustraron aquellas nociones susceptibles de ser complementados con características no instrumentales de las NTIC, y que fueran fundamentales en el presente trabajo. Además, se analizó y destacó que la identidad y visibilidad que proporcionan las NTIC tributan a la seguridad del individuo. Por otro lado, la desterritorialidad y lo translocal implícito en las NTIC deben tenerse en cuenta cuando se estudie la comunicación en una comunidad determinada.

La comunicación social, en el caso tratado de la NTIC, se relaciona con casi todo lo no instrumental estudiado y en particular, con la socialización que es inherente a las NTIC.

Por último creemos necesario expresar que hoy en América Latina, además de las brechas de pobreza y acceso al conocimiento se abre y profundiza la brecha digital. Los Info-ricos y los Infopobres son ahora las nuevas clases, se trata de la abismal distancia que se establece entre los ciudadanos, entre los que acceden a Internet y los que no, entre los que pueden tener un celular y los que no. Esa brecha genera ahora una nueva disfunción: los analfabetos tecnológicos, que supone una discapacidad igual o peor que la de no saber leer y escribir. Ese es el contexto real en el que hemos hecho esta investigación.

Agradecimientos: a Alibeit Kakes y Orieta Alvarez por sus útiles enseñanzas y reflexiones, a Christian Queluz, por invitarnos a presentar este artículo en la Revista Tecnología y Sociedad.

\section{Referencias}

\footnotetext{
${ }_{1}^{1}$ Aguado, Juan Miguel e Martínez, Inmaculada J. (2006) "El proceso de mediatización de la telefonía móvil: de la interacción al consumo cultural".

${ }^{2}$ Geser, Hans. (2004) "The innovative potential of cell phone technology in an evolutionary perspective".

${ }^{3}$ Mattinen, M. (2002). The Messaging Portfolio. Radiolinja Sweden.

${ }^{4}$ Fortunati, L. y Manganelli, A. (2002), "El teléfono móvil de los jóvenes", Revista de Estudios de la Juventud, núm. 57, 2020, Madrid, pp. 59-78.

${ }^{5}$ Ling, R. (2002), "Chicas adolecentes y jóvenes adultos varones: dos subculturas del teléfono móvil", Revista de Estudios de la Juventud, núm. 57, 202, Madrid, pp. 33-46.

${ }^{6}$ Gergen K. J. (2002); Self and Community in the New Floating Worlds. Publicado en:Draft for Nyiri, K. (Ed.) (2002) Mobile democracy, essays on society, self and politics. Vienna:Passagen. 103-114.

7 (Gergen, 2002; Geser, 2004, (Haddon, L. (2000). The social consequences of mobile telephony: Framing questions. Presentado en Sosiale Konsekvenser av Mobiltelefony: Framing. Oslo http://www.telenor.no/fou/prosjekter/Fremtidens Brukere/seminarer/mobilpresentasjoner/Proceedings\%20 FoU\%20notat .pdf , Noruega, junio 2000);( ARAUJO DE SOUZA, A. (2004). From Multiuser Environments as (Virtual) Spaces to (Hybrid) Spaces as Multiuser Environments: Nomadic Technology Devices and Hybrid Communication Places. Tesis Doctoral inédita, presentada en la Universidad Federal de Río de Janeiro.)

${ }^{8}$ Sampedro, Víctor y Sánchez Duarte, José Manuel "La Red era la plaza" En imprenta. Mayo 2011.

${ }^{9}$ Aguado, Juan Miguel e Martínez, Inmaculada J. (2006) "El proceso de mediatización de la telefonía móvil: de la interacción al consumo cultural".

${ }_{10}$ Cabrera Paz, J. (2009), "Pensar lo contemporáneo: de la cultura situada a la convergencia tecnológica”, en Miguel Ángel Aguilar, Eduardo Nivón, María Ana portal, Rosalía Winocur (coordinadores), Barcelona, Anthropos/UAM I.

${ }^{11}$ Sassen, S (2006) "Hay que inventor una Nova Internet", El Jornal de la Ciencia, Año XXI, núm. 581 pp. 5 y 10, 18 de agosto, Sociedad Brasilera para o Progreso da Ciencia.

${ }^{12}$ Ling, R. (2002), "Chicas adolecentes y jóvenes adultos varones: dos subculturas del teléfono móvil", Revista de Estudios de la Juventud, núm. 57, 202, Madrid, pp. 33-46.
} 
${ }^{13}$ Cabrera Paz, J. (2001), "Náufragos y navegantes en territorios hipermediales: experiencias psicosociales y prácticas culturales en la apropiación del internet en jóvenes escolares", Marcelo Bonilla y Gilles Cliché (editores), Internet y sociedad en América Latina y el Caribe, FLACSO Ecuador/ IDRC.

${ }^{14}$ Martín, M. V. (2006), "Jóvenes, identidad y telefonía móvil: algunos ejes de reflexión", Tercer Congreso Online Observatorio para la Cibersociedad www.cibersociedad.net

${ }^{15}$ Thompson (1998), Los Media y la modernidad, Una teoría de los medios de comunicación.

${ }^{16}$ Lévy, P. (1999) ¿Qué es lo virtual?, Buenos Aires, Paidós Multimedia.

${ }^{17}$ Ver: Aguado, J, y Martínez, I. (2006), "El proceso de materialización de la telefonía móvil: de la interacción al consumo cultural", Revista Zer, pp. 319-343. 